\title{
openheart High-pitch versus conventional cardiovascular CT in patients being assessed for transcatheter aortic valve implantation: a real-world appraisal
}

\author{
Tevfik F Ismail, ${ }^{1}$ Emma Cheasty, ${ }^{2}$ Laurence King, ${ }^{3}$ Sahar Naaseri, ${ }^{2}$ Olga Lazoura, ${ }^{2}$ \\ Natalie Gartland, ${ }^{2}$ Simon Padley, ${ }^{2,4}$ Michael B Rubens, ${ }^{2,4}$ Isabel Castellano, ${ }^{3,4}$ \\ Edward D Nicol ${ }^{2,4}$
}

To cite: Ismail TF, Cheasty E, King L, et al. High-pitch versus conventional cardiovascular CT in patients being assessed for transcatheter aortic valve implantation: a realworld appraisal. Open Heart 2017;4:e000626. doi:10.1136/ openhrt-2017-000626

$\mathrm{TI}$ and $\mathrm{EC}$ contributed equally and are joint first author

Received 17 March 2017 Revised 19 April 2017 Accepted 13 June 2017

\section{CrossMark}

${ }^{1}$ Division of Imaging Sciences and Biomedical Engineering, King's College London, London, UK

${ }^{2}$ Radiology Department, Royal Brompton Hospital, London, UK ${ }^{3}$ Medical Physics Department, Royal Marsden Hospital, London, UK

${ }^{4}$ Department of Medicine, Imperial College London, London, UK

Correspondence to Dr Edward D Nicol; e.nicol@ rbht.nhs.uk

\section{ABSTRACT}

Objective High-pitch protocols are increasingly used in cardiovascular CT assessment for transcatheter aortic valve implantation (TAVI), but the impact on diagnostic image quality is not known.

Methods We reviewed 95 consecutive TAVI studies: 44 (46\%) high-pitch and 51 (54\%) standard-pitch. Single high-pitch scans were performed regardless of heart rate. For standard-pitch acquisitions, a separate CTaortogram and CT-coronary angiogram were performed with prospective gating, unless heart rate was $\geq 70$ beats/ min, when retrospective gating was used. The aortic root and coronary arteries were assessed for artefact (significant artefact $=1$; artefact not limiting diagnosis=2; no artefact=3). Aortic scans were considered diagnostic if the score was $>1$; the coronaries, if all three epicardial arteries scored $>1$.

Results There was no significant difference in diagnostic image quality for either the aorta (artefact-free high-pitch: $31(73 \%)$ scans vs standard-pitch: $40(79 \%), p=0.340)$ or the coronary tree as a whole (10 (23\%) vs $15(29 \%)$, $\mathrm{p}=0.493)$. However, proximal coronary arteries were less well visualised using high-pitch acquisitions $(16(36 \%)$ vs $30(59 \%), p=0.04)$. The median (IQR) radiation dose was significantly lower in the high-pitch cohort (dose-length product: 347 (318-476) vs 1227 (1150-1474) mGy cm, respectively, $p<0.001$ ), and the protocol required almost half the amount of contrast.

Conclusions The high-pitch protocol significantly reduces radiation and contrast doses and is non-inferior to standard-pitch acquisitions for aortic assessment. For aortic root assessment, the high-pitch protocol is recommended. However, if coronary assessment is critical, this should be followed by a conventional standard-pitch, low-dose, prospectively gated CT-coronary angiogram if the high-pitch scan is non-diagnostic.

\section{INTRODUCTION}

Transcatheter aortic valve implantation (TAVI) is increasingly being performed as an alternative to conventional aortic valve replacement surgery, particularly for those who have a high surgical risk. ${ }^{12}$ However, the percutaneous devices used for TAVI are only

\section{KEY QUESTIONS}

What is already known about this subject? Gated cardiovascular CT can be used to evaluate the aorta and the coronary arteries in patients being considered for transcatheter aortic valve implantation. It is not known whether newer high-pitch acquisition protocols perform as well as standard-pitch approaches for coronary and aortic evaluation.

\section{What does this study add?}

A high-pitch, dual-source acquisition provides comparable diagnostic information and image quality to a standard-pitch approach with respect to the aorta and the whole coronary tree, but with significantly less radiation and contrast exposure. However, if information is required about the proximal coronary vessels, a standard-pitch protocol is preferable.

How might this impact on clinical practice? For aortic root assessment, the high-pitch protocol is recommended. However, if coronary assessment is critical, this should be followed by a conventiona standard-pitch, low-dose, prospectively gated CTcoronary angiogram if the high-pitch scan is nondiagnostic.

available in a restricted range of sizes. ${ }^{34}$ With conventional surgery, the operator can size the valve prosthesis by directly measuring the aortic root. However, for TAVI, the operator is reliant on preoperative or intraoperative imaging to assess the suitability of the patient and guide device selection. ${ }^{35-7}$ Incorrect device sizing can result in periprocedural complications, including significant paravalvular aortic regurgitation, which has been associated with adverse outcomes in this highrisk cohort. ${ }^{8}$

Gated multidetector CT-cardiovascular angiography (CTCA) allows the reproducible non-invasive assessment of the aortic root, access routes and the distance to the 
coronary ostia. ${ }^{36}$ However, patients being considered for TAVI often have significant coronary artery disease, which may impact on outcomes, ${ }^{9}$ and can also potentially be simultaneously assessed by CTCA. High-pitch, dualsource protocols are increasingly being used in CTCA to minimise radiation dose $\mathrm{e}^{10} 11$ and iodine-based contrast medium load, but it is unclear whether they perform as well as standard-pitch prospective or retrospective ECG-gated protocols for aortic and coronary evaluation in this cohort. We compared diagnostic image quality with respect to the aorta and coronary arteries obtained using a single-acquisition, high-pitch combined wholebody angiogram and gated CT-aortogram/CT-coronary angiogram protocol against a conventional protocol with standard pitches involving a separate non-ECG-gated CT whole-body angiogram and an ECG-gated CT-aortogram/coronary angiogram in patients being considered for TAVI. The protocol evaluation was performed as part of an ongoing service development and improvement programme.

\section{METHODS}

We retrospectively assessed 95 consecutive studies performed for TAVI assessment. Midway through this period, our institution switched from using two acquisitions with standard pitches to obtain a separate whole-body angiogram and gated CT-coronary angiogram/aortogram, respectively, to a high-pitch protocol involving a single acquisition combined CT whole-body angiogram and coronary angiogram/aortogram for imaging TAVI patients. All patients gave written consent for the use of their images for audit and clinical research. The requirement for specific formal ethical approval was waived by the local research and development office as the study involved retrospective review of image quality for studies performed purely for clinical purposes.

\section{Image acquisition}

All images were acquired using a dual-source multidetector scanner (SOMATOM Definition Flash, Siemens, Erlangen, Germany). Neither beta-blockers nor glyceryl trinitrate was administered to any patients in view of their underlying severe aortic stenosis. Patients were scanned with either a single-acquisition high-pitch protocol or with two standard-pitch protocols. For the high-pitch protocol, a single contrast-enhanced acquisition was performed at $80 \mathrm{kV}$ (for patients with a body mass index $(\mathrm{BMI})<20 \mathrm{~kg} / \mathrm{m}^{2}$ ) and at $100 \mathrm{kV}$ or $120 \mathrm{kV}$ (for BMI $>30 \mathrm{~kg} / \mathrm{m}^{2}$ ) from the circle of Willis to the lesser femoral trochanter to allow simultaneous assessment of the aorta, coronary arteries and access routes, with ECG gating used to time the acquisition over the thoracic aortic section at $70 \%$ R-R. For the standard-pitch group, a conventional helical CT whole-body angiogram was performed at $120 \mathrm{kV}$ from the circle of Willis to the lesser trochanter to assess access routes, followed by a second gated acquisition of the heart and thoracic aorta at 80
$\mathrm{kV}$ (for patients with a BMI $<20 \mathrm{~kg} / \mathrm{m}^{2}$ ) and at $100 \mathrm{kV}$ or $120 \mathrm{kV}$ (for BMI $>30 \mathrm{~kg} / \mathrm{m}^{2}$ ). For the latter, patients were scanned with a prospective gating technique with full tube current applied at $70 \%$ of the cardiac cycle for stable heart rates $\leq 60$ beats $/ \mathrm{min}$, or at $60 \%-80 \%$ of the cardiac cycle for unstable heart rates $\leq 60$ beats $/ \mathrm{min}$, or at $30 \%-80 \%$ for heart rates of $61-70$ beats $/ \mathrm{min}$. In patients with significant heart rate variability or heart rates $>70$ beats $/ \mathrm{min}$, retrospective gating with dose modulation was used.

For all patients, a biphasic injection protocol was used. The contrast circulation time was determined using a $15 \mathrm{~mL}$ test bolus, followed by a $40 \mathrm{~mL} 0.9 \%$ saline chaser. The delay time for coronary imaging was the time of peak attenuation in the aortic root plus $3 \mathrm{~s}$ for the standard-pitch protocols or plus $8 \mathrm{~s}$ for the high-pitch protocols. For patients being imaged with the high-pitch protocol, an $80 \mathrm{~mL}$ bolus of contrast (iopromide $370 \mathrm{mg}$ iodine $/ \mathrm{mL}$; Ultravist 370, Bayer Healthcare, Berlin, Germany) was given at $6 \mathrm{~mL} / \mathrm{s}$, followed by a $0.9 \%$ saline chaser of $40 \mathrm{~mL}$ at $6 \mathrm{~mL} / \mathrm{s}$ via a dual-headed injection pump (Stellant D; Medrad UK, Strawberry Hill, Berkshire, UK). For patients imaged with the standard-pitch approach, the whole-body angiogram to assess access routes was performed with a $90 \mathrm{~mL}$ contrast bolus injected at $6 \mathrm{~mL} / \mathrm{s}$, followed by $40 \mathrm{~mL} 0.9 \%$ saline at $6 \mathrm{~mL} / \mathrm{s}$. Subsequently, the CT-coronary angiogram was performed with a $60 \mathrm{~mL}$ contrast bolus at $6 \mathrm{~mL} / \mathrm{s}$, followed by $40 \mathrm{~mL} 0.9 \%$ saline at $6 \mathrm{~mL} / \mathrm{s}$.

\section{Image analysis}

All images were analysed by two experienced cardiovascular imagers using commercially available software (Aquarius, TeraRecon, San Mateo, California, USA). Images were reconstructed using $0.75 \mathrm{~mm}$ sections every $0.6 \mathrm{~mm}$ with a B26f convolution kernel. For retrospectively gated studies, phases were reconstructed at $10 \%$ intervals from $10 \%$ to $100 \%$ of each cardiac cycle. Axial and orthogonal reconstructions of the aortic root and curved multiplanar reformats of the epicardial coronary arteries were assessed for artefact using a 3-point scale (1=artefact significantly limiting diagnosis; $2=$ minimal artefact without diagnostic impact; $3=$ no artefact). For coronary assessment, scans were considered diagnostic if all three epicardial coronary arteries had a score $>1$. Mean contrast enhancement was also assessed at the level of the aortic root and in the distal aorta at the level of the aortic bifurcation. Contrast to noise ratio (CNR) at each location was calculated using:

$$
C N R=\frac{R_{\text {lumen }}-R_{\text {lackground }}}{\text { Noise }}
$$

where $\mathrm{ROI}_{\text {lumen }}$ is the mean CT number in Hounsfield units for a region of interest (ROI) in the aortic lumen, and $\mathrm{ROI}_{\mathrm{fat}}$ is the mean CT number of a similarly sized background ROI placed in an area of body fat. Noise was calculated as:

$$
\text { Noise }=\sqrt{\frac{\sigma_{\text {lumen }}^{2}+\sigma_{\text {background }}^{2}}{2}}
$$




\begin{tabular}{|c|c|c|c|}
\hline & $\begin{array}{l}\text { High- } \\
\text { pitch }(n=44)\end{array}$ & $\begin{array}{l}\text { Standard- } \\
\text { pitch }(n=51)\end{array}$ & p Value \\
\hline Male, n (\%) & $24(55)$ & $26(51)$ & 0.837 \\
\hline $\begin{array}{l}\text { Age, median } \\
\text { (IQR) years }\end{array}$ & $83.5(75.5-85.0)$ & $81.0(71.0-86.0)$ & 0.261 \\
\hline $\begin{array}{l}\text { Weight } \\
\text { (mean } \pm S D), k g\end{array}$ & $73.5 \pm 15.5$ & $71.5 \pm 13.0$ & 0.532 \\
\hline $\begin{array}{l}\text { Heart rate, } \\
\text { median }(\mathrm{QQR}) \text {, } \\
\text { per minute }\end{array}$ & $70.5(61.0-80.0)$ & $69.0(63.0-79.0)$ & 0.925 \\
\hline
\end{tabular}

where $\sigma_{\text {lumen }}$ and $\sigma_{\text {background }}$ are the SD of the CT numbers for the same similarly sized ROIs in the aortic lumen and background body fat, respectively. The CNR was measured in this way three times in non-adjacent slices and the mean value used.

The radiation dose for each study was calculated as the total dose-length product (DLP, $\mathrm{mGy} \mathrm{cm}$ ) as previously described, ${ }^{12}$ together with the volume CT dose index $\left(\mathrm{CTDI}_{\mathrm{vol}}\right)$ for the whole-body angiogram. Effective dose for a standard-sized patient was estimated using the ImPACT dose calculator in accordance with International Committee on Radiation Protection (ICRP) $60 .^{13} 14$

\section{Statistical analysis}

The Shapiro-Wilks test was used together with histograms to assess the normality of continuous data. Continuous variables are expressed as mean $\pm \mathrm{SD}$ for normally distributed variables and as medians with IQRs for non-parametric data. Differences between parametric continuous variables were assessed using Student's t-test, and for non-parametric data the Mann-Whitney U test. Categorical data are presented as frequencies and percentages. Differences between categorical variables were assessed using the $\chi^{2}$ and Fisher's exact tests as appropriate. All data were analysed using MedCalc V.13 (MedCalc Software, Mariakerke, Belgium). Two-tailed values of $p<0.05$ were considered statistically significant.

\section{RESULTS}

Overall, 44/95 (46\%) of studies were performed using the high-pitch protocol and the remainder were performed at a standard-pitch using either retrospectively gated or prospectively gated protocols depending on heart rate. There were no significant differences between the groups with respect to gender, age, weight or heart rate (table 1).

The contrast enhancement (mean \pm SD) achieved at the level of the aortic root was comparable for the two cohorts but was significantly higher in the high-pitch cohort in the distal aorta (table 2). The CNR obtained with the standard-pitch approach was statistically significantly higher than that obtained with the high-pitch protocol for both the aortic root and distal aorta (table 2).

With respect to the aortic root, with either protocol, none of the patients had any significant artefact that limited diagnosis, and there was also no statistically significant difference in the frequency of scans without any artefact affecting the aorta (table 2). Examples are illustrated in figure 1 . For the coronary reconstructions, 10 $(23 \%)$ scans in the high-pitch group and 15 (29\%) in the standard-pitch group were considered fully diagnostic $(p=0.493)$. However, when considering only the proximal coronary vessels, there was a significantly higher frequency of diagnostic studies with the standard-pitch protocol (high-pitch: $16(36 \%)$ vs standard-pitch: 30 $(59 \%)$ diagnostic studies, $\mathrm{p}=0.04)$. When analysed by vessel, there was no significant difference between the two protocols for whole coronary tree assessment (table 3). When considering only the proximal vessels, the proximal circumflex was significantly more liable to artefact with the high-pitch versus the standard-pitch acquisition (table 3). Examples of each of the artefact grades are illustrated in figure 2 .

The distributions by weight of total exam DLP and CTDI $_{\text {vol }}$ for the whole-body angiogram are illustrated in figures 3 and 4, respectively. These reveal a linear relationship between both DLP and CTDI ${ }_{\text {vol }}$ and weight for the standard-pitch protocol, reflecting the automatic adjustment of tube current with patient size. In contrast,

\begin{tabular}{|c|c|c|c|}
\hline & High-pitch $(n=44)$ & Standard-pitch $(n=51)$ & p Value \\
\hline \multicolumn{4}{|c|}{ Contrast enhancement (mean $\pm S D$ ), Hounsfield units } \\
\hline Aortic root & $455 \pm 161$ & $464 \pm 123$ & 0.760 \\
\hline Distal aorta & $491 \pm 193$ & $362 \pm 74$ & $<0.001$ \\
\hline \multicolumn{4}{|c|}{ Contrast to noise ratio (mean $\pm S D$ ) } \\
\hline Aortic root & $18.0 \pm 3.98$ & $26.1 \pm 6.52$ & $<0.001$ \\
\hline Distal aorta & $14.2 \pm 5.72$ & $16.7 \pm 4.93$ & $<0.001$ \\
\hline \multicolumn{4}{|c|}{ Aortic artefact grade, $\mathrm{n}(\%)$} \\
\hline 1 & $0(0)$ & $0(0)$ & 0.340 \\
\hline 2 & $13(29.5)$ & $11(21.36)$ & \\
\hline 3 & $31(70.5)$ & $40(78.4)$ & \\
\hline
\end{tabular}



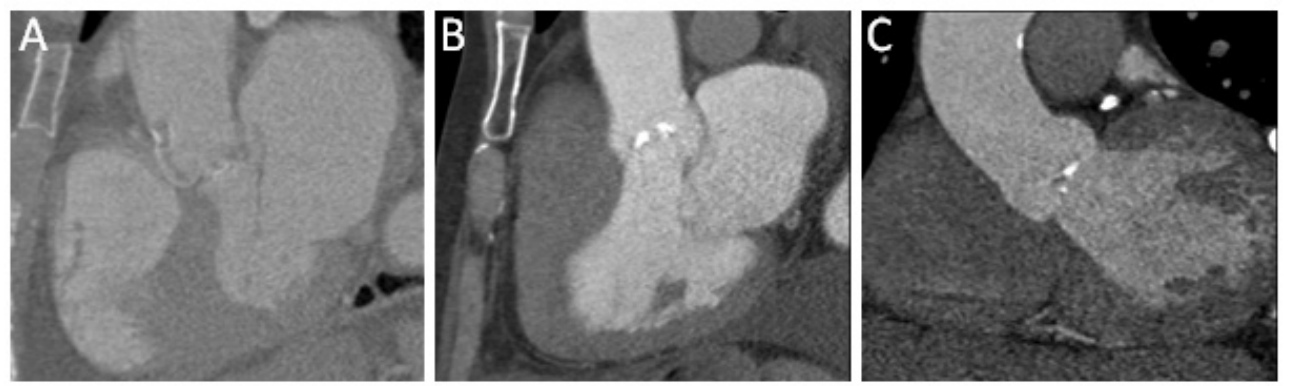

Figure 1 Examples of CT-aortograms illustrating the grading system used to evaluate diagnostic image quality. (A) Significant artefact=1; (B) artefact not limiting diagnosis=2; (C) no artefact=3.

both DLP and CTDI ${ }_{\text {vol }}$ rise in a stepwise fashion with weight for the high-pitch protocol, indicating that the prescribed tube current would exceed the maximum available and is therefore capped; the increase in dose is therefore simply due to the change in tube voltage with BMI. The median (IQR) total exam DLP was significantly lower in the high-pitch group than the standard-pitch group (high-pitch: 347 (318-476) vs standard-pitch: 1227 (1150-1474) mGy cm, p<0.001). Similarly, the median (IQR) CTDI ${ }_{\text {vol }}$ for the whole-body acquisition was significantly lower in the high-pitch versus the standard group (high-pitch: 3.60 (3.34-3.79) vs standard-pitch: 8.96 (8.03-11.4) $\mathrm{mGy}, \mathrm{p}<0.001)$. The effective dose for a standard-sized patient, estimated using the ImPACT dose calculator in accordance with ICRP 60 , was $6 \mathrm{mSv}$ for the high-pitch protocol and ranged between 13 and $29 \mathrm{mSv}$ for the standard-pitch protocol depending on the gating technique used. The iodine-based contrast medium load required for the high-pitch studies was $80 \mathrm{~mL}$ vs $150 \mathrm{~mL}$ in the standard-pitch study.

Table 3 Coronary artefact grades for the high-pitch versus standard-pitch groups broken down by whole epicardial coronary artery and proximal coronary artery

\begin{tabular}{|c|c|c|c|}
\hline & $\begin{array}{l}\text { High-pitch } \\
(n=44)\end{array}$ & $\begin{array}{l}\text { Standard-pitch } \\
(n=51)\end{array}$ & p Value \\
\hline Left anterior descending, $\mathrm{n}(\%)$ & & & 0.545 \\
\hline 1 & 14 & 13 & \\
\hline 2 & 15 & 23 & \\
\hline 3 & 15 & 15 & \\
\hline Left circumflex artery, $\mathrm{n}(\%)$ & & & 0.089 \\
\hline 1 & 24 & 18 & \\
\hline 2 & 13 & 16 & \\
\hline 3 & 7 & 17 & \\
\hline Right coronary artery, $\mathrm{n}(\%)$ & & & 0.927 \\
\hline 1 & 22 & 26 & \\
\hline 2 & 12 & 15 & \\
\hline 3 & 10 & 10 & \\
\hline Proximal left anterior descending, $\mathrm{n}(\%)$ & & & 0.402 \\
\hline 1 & 6 & 3 & \\
\hline 2 & 11 & 12 & \\
\hline 3 & 27 & 36 & \\
\hline Proximal left circumflex artery, $\mathrm{n}(\%)$ & & & 0.006 \\
\hline 1 & 18 & 7 & \\
\hline 2 & 12 & 14 & \\
\hline 3 & 14 & 30 & \\
\hline Proximal right coronary artery, n (\%) & & & 0.238 \\
\hline 1 & 18 & 13 & \\
\hline 2 & 10 & 12 & \\
\hline 3 & 16 & 26 & \\
\hline
\end{tabular}



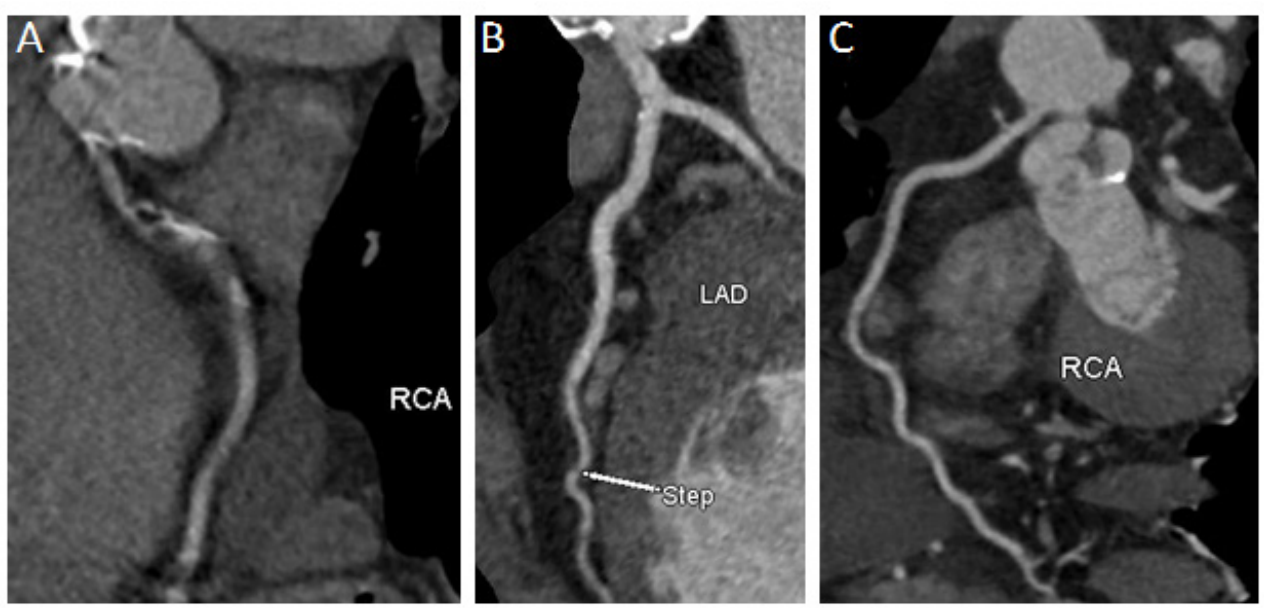

Figure 2 Representative CT-coronary angiogram curved multiplanar reformats illustrating grading system used to evaluate diagnostic image quality. (A) Significant artefact=1; (B) artefact not limiting diagnosis=2; (C) no artefact=3. LAD, left anterior descending; RCA, right coronary artery.

\section{DISCUSSION}

The use of a high-pitch versus standard-pitch protocol significantly reduces radiation and iodine-based contrast medium load, while achieving comparable diagnostic image quality both with respect to the aorta and the whole coronary tree in patients being assessed for TAVI. However, a high-pitch acquisition is not sufficient if evaluation of the proximal coronaries is required.

Patients being considered for TAVI often undergo numerous investigations to assess their suitability for the procedure. ${ }^{37}$ These focus on the measurement of aortic root dimensions to facilitate device selection and assessment of potential access routes for the procedure. ${ }^{6}$ Although other modalities such as cardiovascular magnetic resonance (CMR) can provide comparable information, ${ }^{3}$ a significant number of patients have implanted devices that contraindicate this, or experience difficulty with lying flat in the scanner for significant lengths of time.

Cardiovascular CT offers the versatility of being able to assess access routes and aortic root dimensions in a single study. ${ }^{6}$ In contrast to CMR, it also has the temporal and

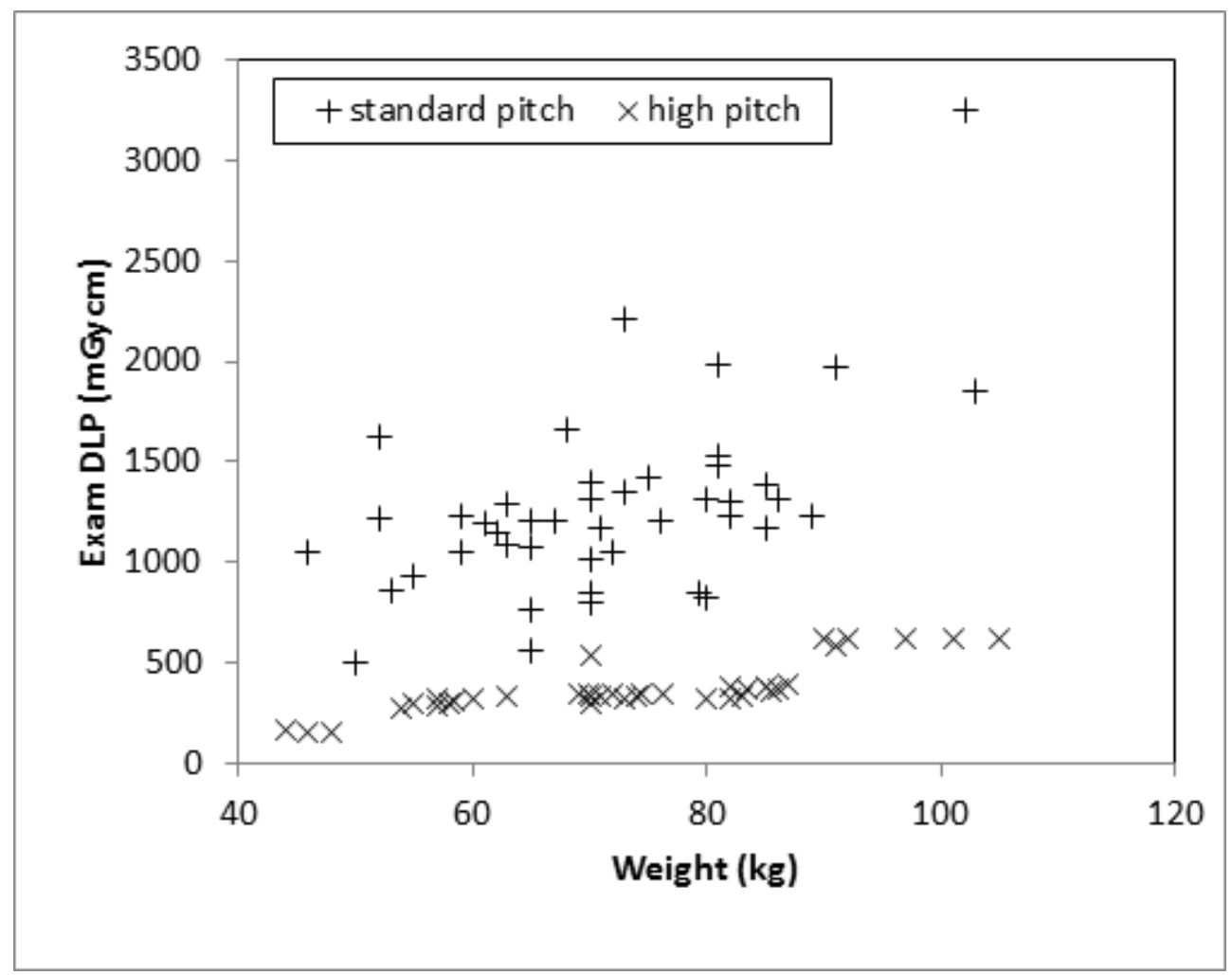

Figure 3 Total exam dose-length product (DLP) for the high-pitch and standard-pitch protocols against weight. 


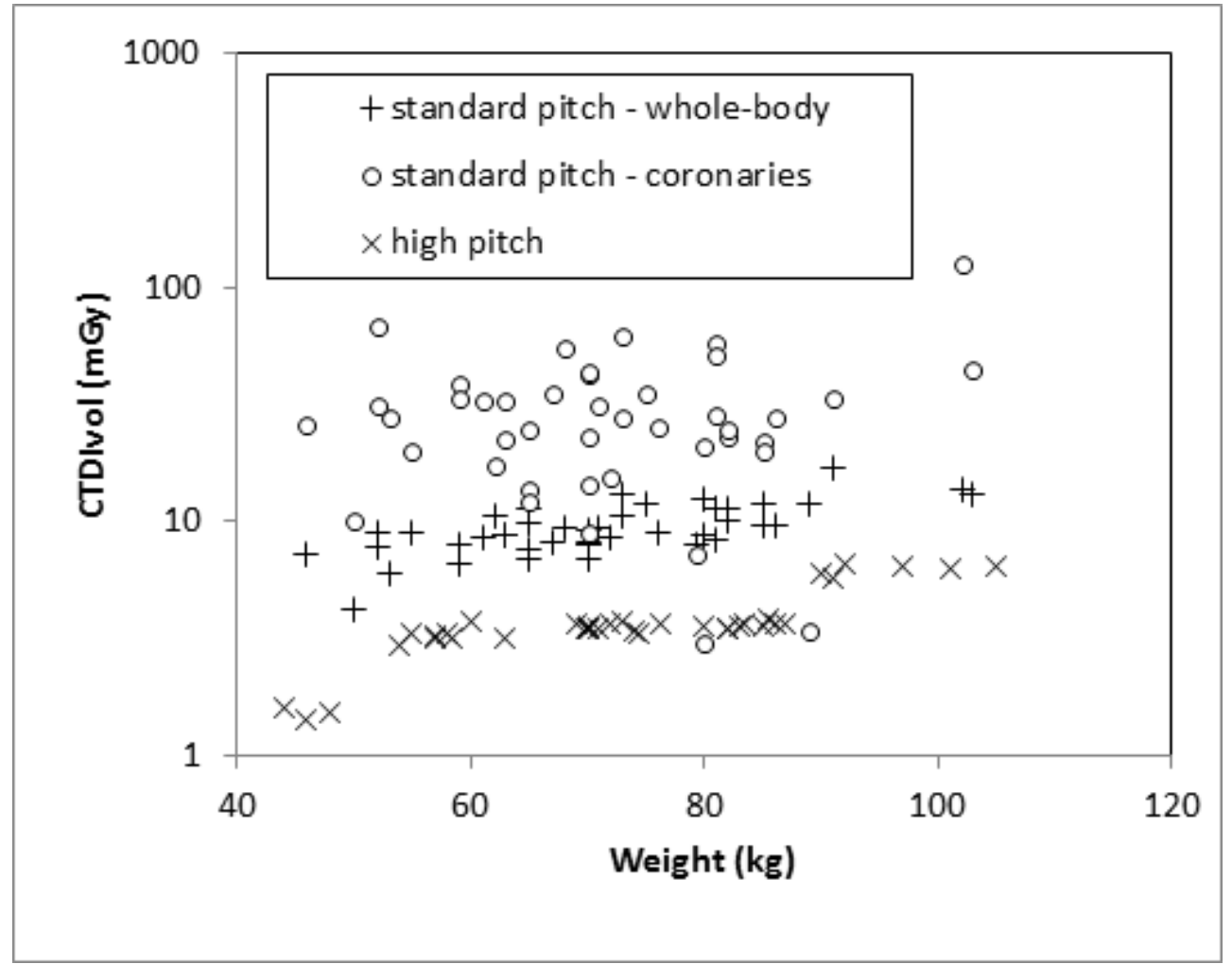

Figure 4 CT dose-index (CTDI $\left.{ }_{\text {vol }}\right)$ for the high-pitch protocol and standard-pitch whole-body angiogram and coronary protocols against weight.

spatial resolution to be potentially able to simultaneously assess the coronary arteries. This represents a significant advantage over other modalities as the height of the coronary ostia from the aortic valve annulus can be readily determined by CT and may significantly impact on the safety of device deployment. ${ }^{4}$ It is therefore an important consideration when determining the appropriateness of TAVI. Cardiovascular CT can also potentially allow the concomitant detection of significant coronary disease, which may increase the risks of ischaemia during the procedure, particularly during the rapid ventricular pacing required immediately prior to device deployment. Given the high burden of coronary disease in this population, this has traditionally been determined by invasive coronary angiography. The latter imposes an additional contrast load, and for those who ultimately prove unsuitable for TAVI, exposure to the risks of the invasive procedure with minimal gain. It would therefore be desirable to obviate the risks of this additional invasive assessment using CT-coronary angiography. Such an approach has been successfully used in other patient groups undergoing non-coronary cardiothoracic surgery $^{15}$ and in other settings where it is desirable to obviate invasive assessment. ${ }^{16}$

We found that gating the acquisition of the CT-aortogram undertaken as part of TAVI assessment regardless of which protocol was used produced comparable results in terms of aortic assessment. None of the patients in either the high-pitch or standard-pitch groups experienced any artefact that significantly affected image quality when assessing the aorta. We also found that with the use of a test bolus to optimise the timing of contrast delivery and image acquisition, similar degrees of contrast enhancement were obtained in the aortic root irrespective of which protocol was employed. However, the mean CT number for the distal aorta was significantly higher with the high-pitch protocol, reflecting the combined effects of the lower $\mathrm{kV}$ generally employed with this approach, and the greater X-ray attenuation by the denser abdominal viscera versus the less attenuating air-filled lungs in the thorax. The CNR was statistically significantly higher with the standard pitch protocols, again reflecting the generally higher tube potentials employed. These small although statistically significant differences in objective image quality however did not translate into meaningful clinical differences. The mean aortic attenuations and CNR achieved in the high-pitch protocol were higher or comparable to those described by Wuest et $a l,{ }^{17}$ who used a high-pitch only approach, with a similar effective radiation dose. However, the latter study did not make any direct comparisons with standard-pitch approaches or formally evaluate the coronary tree. Nevertheless, the work by Wuest $e t$ al demonstrates that further reductions in contrast dose are possible with high-pitch approaches. ${ }^{17}$

In the present study, there was no significant difference in the subjective diagnostic quality of aortograms obtained by either protocol. Furthermore, motion artefact significantly affected the quality of the CT-coronary angiograms obtained, regardless of protocol, such that over two-thirds were non-diagnostic across the whole 
cohort. The choice of protocol had no impact on the ability to obtain diagnostic image quality with respect to motion artefact when the entire coronary tree was considered. However, the standard-pitch protocol performed significantly better than the high-pitch approach when considering only the proximal segments of the three main epicardial coronary arteries. Disease in the proximal segments may be of greater potential prognostic importance than distal disease, particularly if there is concomitant left ventricle (LV) dysfunction. ${ }^{18}$ Information about these vessel segments alone may therefore retain utility in some clinical settings.

Coronary imaging in this patient cohort is particularly challenging as beta-blockers are contraindicated in severe aortic stenosis, making adequate heart rate control difficult to achieve. The median heart rate for patients in both groups was $\sim 70$ beats/min, significantly higher than the regular 60 beats/min or less normally desirable for coronary CT-angiography. ${ }^{10}$ This reflects the fact that with a limited aortic valve area, and therefore stroke volume, such patients maintain their cardiac output by augmenting their resting heart rate. It is likely that this had a greater impact on the frequency of diagnostic scans than the choice of protocol.

Although this was not formally assessed in this study, we have previously found that patients being considered for TAVI have significant coronary calcification in association with their aortic valve disease, which can preclude adequate coronary evaluation by CT-coronary angiography, regardless of image quality. ${ }^{19}$ This is unsurprising given that the majority of these patients are being considered for TAVI rather than conventional surgery for the very reason that they are elderly or have significant comorbidities such as chronic kidney disease, diabetes and coronary disease. Based on these considerations and the low diagnostic yield obtained in the present study, irrespective of the protocol employed, we suggest that CT-coronary angiography can still as yet not routinely be used to evaluate the coronary arteries in patients being considered for TAVI.

However, if information is required about the coronary arteries, particularly the proximal vessel segments, a standard CT-coronary angiogram should be considered, although even with this approach we found that the diagnostic yield was at most 59\%. The high-pitch approach however by definition only provides one single phase for coronary evaluation offering less versatility for coronary evaluation, particularly at more rapid heart rates. More recently, we and others have found that end-systolic imaging $(20 \%-40 \%)$ with our dual-source scanner is adequate for coronary assessment at higher heart rates, ${ }^{20}$ and so the highest dose retrospective acquisitions are no longer performed in our institution.

Although with respect to both the aorta and the coronary arteries we found no significant differences in the rates of diagnostic imaging using the high-pitch or the standard-pitch approaches, the total radiation dose and contrast load with the high-pitch protocols were significantly lower than with the standard-pitch approach. Given their underlying severe aortic stenosis, age and other comorbidities, radiation dose may seem of less relevance to patients being considered for $\mathrm{TAVI}^{6}$; however, iodine-based contrast medium load is important as many patients being considered for TAVI have associated chronic renal impairment. Our data suggest that high-pitch protocols should be considered as part of efforts to adhere to the 'As Low As Reasonably Achievable' principle and to minimise the contrast load used in these patients. While attempts to reduce radiation dose should not be at the expense of image quality, our data suggest that the adoption of high-pitch protocols has the potential to significantly minimise radiation exposure and more importantly contrast load without compromising diagnostic value. With improvements in TAVI techniques and devices, this technique may in future be used for lower risk patients in whom open surgery would have been previously considered. ${ }^{21}$ We speculate that lowering radiation exposure among this cohort may therefore assume greater importance on a population basis as TAVI begins to be offered for younger patients with fewer comorbidities and longer anticipated life expectancy.

\section{Limitations}

Our study has several potential limitations. First, it was retrospective in nature risking selection bias. Second, patients were not randomly assigned to one protocol or another, but the impact of a systematic change in protocol was assessed as part of an ongoing programme of service improvement and protocol development. However, consecutive patients were studied over the period during which the protocol change took place and both groups were comparable with respect to heart rate and demographic factors that may have influenced image quality. Finally, all patients were scanned at a single tertiary centre on the same platform. While this facilitated the use of a standardised approach, it is possible that different results may be obtained on different platforms or with different hardware. Careful evaluation of any new strategy is therefore essential as part of any service improvement or systematic change in scan protocols.

\section{CONCLUSION}

The use of a high-pitch versus standard-pitch imaging protocol did not significantly impact on the ability to obtain diagnostic images with respect to significant motion artefact for either the aorta or whole coronary tree assessment in patients being considered for TAVI. Where available, highpitch techniques should therefore replace standard-pitch approaches for TAVI assessment and will afford significant reductions in radiation and contrast dose, without any significant decrement in diagnostic image quality. The only caveat to this advice is for those patients where concomitant CT assessment of the coronary arteries is critical. In this clinical scenario, we recommend a high-pitch CT-aortogram for 
TAVI assessment, which if not diagnostic for the coronaries should be followed by a standard-pitch, low-dose CT-coronary angiogram, with end-systolic prospective acquisition preferred where available rather than retrospective gating for patients with heart rates $>70$ beats $/ \mathrm{min}$.

Acknowledgements The authors wish to thank the radiographers and the PACS team for their assistance with undertaking this work. None of the authors report any relevant disclosures.

Contributors All authors contributed significantly to the work. TFI, EC and EDN devised and planned the study. TFI and EC performed clinical image analysis. LK and IC performed image noise/CNR evaluation, statistical analysis and dosimetry work. TFI drafted the manuscript. EC, LK, SN, OL, NG, SP, MBR, IC and EDN critically reviewed and revised the manuscript for important intellectual content. EDN was responsible for overall study supervision.

Funding No funding was required for this work.

Competing interests None declared.

Ethics approval Research and Development Office, Royal Brompton Hospital.

Provenance and peer review Not commissioned; externally peer reviewed.

Data sharing statement № additional data are available.

Open Access This is an Open Access article distributed in accordance with the Creative Commons Attribution Non Commercial (CC BY-NC 4.0) license, which permits others to distribute, remix, adapt, build upon this work non-commercially, and license their derivative works on different terms, provided the original work is properly cited and the use is non-commercial. See: http://creativecommons.org/ licenses/by-nc/4.0/

(c) Article author(s) (or their employer(s) unless otherwise stated in the text of the article) 2017. All rights reserved. No commercial use is permitted unless otherwise expressly granted.

\section{REFERENCES}

1. Leon MB, Smith CR, Mack M, et al. Transcatheter aortic-valve implantation for aortic Stenosis in patients who cannot undergo surgery. N Engl J Med 2010;363:1597-607.

2. Hamm CW, Möllmann $\mathrm{H}$, Holzhey $\mathrm{D}$, et al. The erman aortic valve registry (GARY): in-hospital outcome. Eur Heart J 2014;35:1588-98.

3. Jabbour A, Ismail TF, Moat N, et al. Multimodality imaging in transcatheter aortic valve implantation and post-procedural aortic regurgitation: comparison among cardiovascular magnetic resonance, cardiac computed tomography, and echocardiography. $J$ Am Coll Cardiol 2011;58:2165-73.

4. Achenbach S, Delgado V, Hausleiter J, et al. SCCT expert consensus document on computed tomography imaging before transcatheter aortic valve implantation (TAVI)/transcatheter aortic valve replacement (TAVR). J Cardiovasc Comput Tomogr 2012;6:366-80.

5. Zamorano JL, Gonçalves A, Lang R. Imaging to select and guide transcatheter aortic valve implantation. Eur Heart $J$ 2014;35:1578-87.
6. Leipsic J, Gurvitch R, Labounty TM, et al. Multidetector computed tomography in transcatheter aortic valve implantation. JACC Cardiovasc Imaging 2011;4:416-29.

7. Messika-Zeitoun D, Serfaty JM, Brochet E, et al. Multimoda assessment of the aortic annulus diameter: implications for transcatheter aortic valve implantation. J Am Coll Cardiol 2010;55:186-94.

8. Sherif MA, Abdel-Wahab M, Stöcker B, et al. Anatomic and procedural predictors of paravalvular aortic regurgitation after implantation of the Medtronic CoreValve bioprosthesis. J Am Coll Cardiol 2010;56:1623-9.

9. Dewey TM, Brown DL, Herbert MA, et al. Effect of concomitant coronary artery disease on procedural and late outcomes of transcatheter aortic valve implantation. Ann Thorac Surg 2010;89:758-67. discussion 67.

10. St Noble V, Douraghi-Zadeh D, Padley SP, et al. Maximizing the clinical benefit of high-pitch, single-heartbeat CT coronary angiography in clinical practice. Clin Radiol 2014;69:674-7.

11. Achenbach S, Goroll T, Seltmann M, et al. Detection of coronary artery stenoses by low-dose, prospectively ECG-triggered, highpitch spiral coronary CT angiography. JACC Cardiovasc Imaging 2011:4:328-37.

12. Mahoney R, Pavitt CW, Gordon D, et al. Clinical validation of dualsource dual-energy computed tomography (DECT) for coronary and valve imaging in patients undergoing trans-catheter aortic valve implantation (TAVI). Clin Radiol 2014;69:786-94.

13. Keat N. ImPACT - Imaging Performance Assessment of CT Scanners. CT Dosimetry. London: ImPact, 2011.

14. ICRP. Recommendations of the International Commission on Radiological Protection. Pergammon: Oxford, 1991.

15. Bettencourt N, Rocha J, Carvalho M, et al. Multislice computed tomography in the exclusion of coronary artery disease in patients with presurgical valve disease. Circ Cardiovasc Imaging 2009;2:306-13.

16. Andreini D, Pontone G, Pepi M, et al. Diagnostic accuracy of multidetector computed tomography coronary angiography in patients with dilated cardiomyopathy. J Am Coll Cardiol 2007:49:2044-50.

17. Wuest W, Anders K, Schuhbaeck A, et al. Dual source multidetector CT-angiography before transcatheter aortic valve implantation (TAVI) using a high-pitch spiral acquisition mode. Eur Radiol 2012;22:51-8.

18. Caracciolo EA, Davis KB, Sopko G, et al. Comparison of surgical and medical group survival in patients with left main equivalent coronary artery disease. Long-term CASS experience. Circulation 1995;91:2335-44.

19. Snow T, Zielke SW, Brinkert M, et al. Non rate-controlled CT coronary angiography for the exclusion of obstructive coronary artery disease in the assessment of patients referred for transcatheter aortic valve implantation. Eur Heart $J$ 2013;34:P3082.

20. Goetti R, Feuchtner G, Stolzmann P, et al. High-pitch dual-source CT coronary angiography: systolic data acquisition at high heart rates. Eur Radiol 2010;20:2565-71.

21. Wenaweser P, Stortecky S, Schwander S, et al. Clinical outcomes of patients with estimated low or intermediate surgical risk undergoing transcatheter aortic valve implantation. Eur Heart $J$ 2013;34:1894-905. 